\title{
Objective Testing for Collage Translation Course and Its Washback Effects
}

\author{
$\mathrm{Ma} \mathrm{Li}$ \\ School of Foreign Languages, East China University of Political Science and Law, Shanghai, China (ml0088@ 126.com)
}

\begin{abstract}
The traditional test for translation course is mainly based on the subjective test, being composed of text translations. However the contemporary teaching syllabus and the content of the translation course cover wide range of theories and techniques in translation apart from the text translations. Therefore the subjective test based on the text translations is not adequate in measuring the achievement of teaching aim and the students' command of the course content. Meanwhile the labor cost and the personal factors from the examinees involved in the evaluation process also call for the multiplied test genres for translation. The paper tends to explore the feasibilities in objective testing for translation course and its feedbacks.
\end{abstract}

Keywords-- translation course, objective testing, washback effects

\section{大学翻译课程的客观测试及反拨效应}

\author{
马莉 \\ 华东政法大学外语学院, 上海, 中国
}

\begin{abstract}
摘 要 传统的大学翻译课程测试是双语互译, 以主观试题为主。但现阶段的翻译课程大纲和课程内容却涵盖了除文本翻译外的 大量理论和技巧。因此, 仅仅以文本翻译为内容的主观试题未能考查教学目的是否达到、考生对课程内容是否掌握的作用。同时, 评 判文本翻译时的人力投入及评判者的主观因素都构成了在翻译课程测试中对多元化题型的需求。本文探讨的是客观试题在翻译测试中 的可行性及对教学的反拨作用。
\end{abstract}

关键词 翻译课程, 客观测试, 反拨效应

\section{1. 引言}

翻译在我国高等院校外语院系的课程名称通常为《翻 译理论与实践》, 是外语专业高年级的一门必修课, 也是专 业主干课程。《高等学校英语专业英语教学大纲》[6]对翻 译教学的要求是:初步了解翻译基础理论和英、汉两种语言 的异同,并掌握常用的翻译技巧。能运用翻译的理论和技巧, 将英美报刊上的文章以及文学原著译成汉语, 或将我国报 刊上的文章和一般文学作品译成英语。译文要求忠实原意, 语言流畅。这一教学要求同时也为我们确立了基本的教学 目标, 即: 了解英汉两种语言的差异; 熟悉基本翻译技巧; 掌握新闻及文学等文体的不同翻译特点。随着我国加入 WTO, 翻译市场的潜力不断增大, 在日常外事、经济、科技、 贸易、法律等领域对翻译人才的需求也急剧增长。毫无疑

国家社会科学基金项目支持（资助号：12BYY019）
问, 在英语专业各项技能听、说、读、写、译中, 翻译是 体现语言应用能力的重要标准。

\section{2. 翻译测试的现状}

测试的目的则是提供一种科学的测试工具, 通过对学 生语言运用能力的抽样测试, 对学生的语言能力进行客观 的、准确的、公正的评价。

对于翻译测试, 传统的理解往往顾名思义, 即以两种 语言的文本语码转换为主要依据。据穆雷在进行“中国翻译 教学研究”的项目研究中, 调查了我国部分高校翻译测试的 题型,结果是: $20 \%$ 的学校翻译测试只是一篇短文翻译,50\% 的学校除句子翻译和短文翻译外, 另有回答问题或改错等 题型, 但一般不超过三种题型, 只有 $30 \%$ 的学校出题时有五 种以上的题型,包括单词翻译、单句翻译、短语翻译或成语 翻译、短文翻译、回答问题、选择填空或改正错误及译评 
等内容。[7]与此同时, 翻译或写作类的测试都以繁重的阅 卷工作量而使广大教师望而却步。

一门课程的测试是衡量学生学业成就的主要工具。是 检查教学大纲执行情况、评估教学质量、促进教学改革的 重要手段。鉴于翻译课程的教学目标和课程内容, 只测试 单纯的句子、段落的翻译显然不能起到考查教学目的是否 达到、考生对课程内容是否掌握的作用, 也就是说试题缺 乏表面效度或内容效度, 也是一般学业成就测试 （Achievement Test）无效的原因。这种过于简单的命题方 式的缺陷是, 测试目的不明确, 未能覆盖课程内容的几大知 识模块, 试题内容难以涉及到需要检查的两种语言的理论 知识、翻译技巧和技能。

全国英语专业八级考试( TEM8) 的翻译测试便将这些 要考的能力和教学大纲的要求细化为测试操作的六个方面: (1)根据主题、文体、场合、情景和读者的不同,采用不同的 翻译方法; (2)流利、准确地表达原语的思想涵义;(3)忠实地 传达原语的信息; (4)理解直译和意译的不同; (5) 解翻译的 基本技巧; (6)了解英语和汉语在使用、词序、语法结构和修 辞方法上的不同。[9]要体现上述种种能力, 绝非单一的一 篇英汉语篇翻译可概括, 但由于诸多原因所限, 八级翻译 测试的英译汉和汉译英所选择的题型是各自一篇短文翻译, 虽然其效度也得到了肯定, 但显然未能全方位地考察学生 的理论功底和系统知识, 可以说, 在此阶段的此种考核方 式, 主要证明的是学生两种语言的基础水平, 对翻译课程 本身的设置、建设和发展方向没有直接启示作用。

从《高等学校英语专业英语教学大纲》对翻译教学的 要求可以看出, 理解能力和表达能力是测试的要素, 同时要 兼顾考查学生对翻译基础理论、翻译技巧和英汉两种语言 及文化对比知识的掌握和运用。翻译的测试只有体现了上 述的能力, 方能体现翻译的结构效度。因此, 测试的内容 及形式必须以要考查的能力为出发点, 以实现考试的“结构 效度”为目的。

\section{3. 翻译测试中客观试题的反拨效应}

教育界把测试对教学和学习的影响称为反拨效应。后 来, 英国应用语言学家把这一概念引用到应用语言学领域, 特指语言测试、尤其是外语测试对相应的教学和学习产生 的影响。测试的反拨效应可以是正面的，也可能是负面的。 对测试的反拨效应研究的主要目的之一就是探索减少负面 反拨效应、提高正面反拨效应的途径。Hughes提出了提高 测试正面反拨效应的七点意见: 测试要培养能力测试; 内 容覆盖面要广并具有不可预测性; 使用直接测试; 使用尺 度参照; 成就测试要基于教学目标; 使学生和教师了解测 试; 必要时向教师提供协助。[9]
同时, Hughes也认识到, 要完全做到以上几点, 特别是 直接测试, 需要高昂的成本, 是违背了测试实用性的原则 的。因此, 我们可以看到学者们在对测试的信度与效度研 究的同时, 也将的测试所要花费的成本纳入试题研发的范 围, 引发了我们对主观题型和客观题型的探讨。

\section{1 客观测试在翻译测试中的可行性}

客观测试是全国大规模考试所普遍采用的一种形式。客 观测试的理论来源于结构主义语言学的理论。结构主义认 为语言是由很多小结构构成的。语言中的语音、词汇、语 法构成了一个系统, 这一系统是可以分解的。因此, 掌握一门 语言就是掌握这门语言中的这些元素并用它们来生成和理 解无限数量的句子的能力。由于语言是可以分解成构成元 素的,因此可以设计出离散的题目来逐项测验学生是否掌 握了这些元素。从心理测量的角度来说,任何测试只可能是 一种随机采样, 在有限的时间内不可能对构成语言的全部 元素逐一进行测验, 可通过随机采样对全部构成元素的一 部分进行测验,然后推断对构成元素全体的掌握程度。客观 测试的形式有多种, 如多项选择、正误选择、完形填空、 对应词选择、词义分类等, 最典型的代表是多项选择题 (MC) 。

以客观类题型为主要测试手段的大学英语四级、六级 考试近年来引起了人们广泛的争议, 有人认为学生可靠猜 题来获取分数, 因此对客观题型的信度、效度提出质疑。 而对于翻译和写作的测试方式, 更是主观题型的一统天下, 客观试题无论在理论上和实践中都基本不占一席之地。那 么客观测试到底能否科学地、有效地测量出学生在翻译中 的学习和运用能力? 在翻译课程的教学中如何正确地使用 客观测试? 在此笔者试图从设计、开发语言测试一般要遵 循的 2 个原则———致性原则和有用性原则来解答。

语言测试的第一个原则是一致性原则。Bachman和 Palmer认为语言测试设计、开发语言测试的真实性指目标 语言使用任务特征与测试任务特征的一致性。测试任务与 被试平时使用的语言或内容越接近, 其测试行为就会发挥 得越好, 测试的真实性就越强。[1]传统翻译测试中的单词 翻译、单句翻译、短语翻译或成语翻译、短文翻译、回答 问题等主观题型所考察的只是文本翻译实践, 对教学内容 的覆盖很有限。诚然, 在简答题中可考核翻译理论及技巧 的知识, 但笔者认为假设目的为考核学生的翻译理论及技 巧知识, 此种题型弊端有二: 一是学生的表述方式与书写 风格不尽相同, 毫无疑问会影响到评阅者的认同程度。而 客观测试的答案只有一个,阅卷人员只能按照唯一的正确 答案来评分, 阅卷人员的喜好、兴趣和风格不能影响评卷成 绩。二是在客观试题可达到同样效果的情况下, 该种评阅 
方式无端加重了教师的阅卷负担。

语言测试的第二个原则是有用性原则。Bachman认为 语言测试的有用性主要包括 4 个方面的特征, 即: 信度 (reliability) 、效度(validity) 、可行性(practicality) 、影响 (impact)。[2]信度和效度是一项测试的最重要因素。信度是 指对学生的语言水平提供可靠的度量; 效度指考试能够准 确地反映学生实际运用语言的能力。可行性指从人力、物 力、时间上测试是否得以实施, 是否可行。可行性主要与实 施测试的方式以及在多大程度上要建立和采用某项测试有 关。客观测试的可行性较强, 并且客观测试抽样量大, 覆盖 面宽, 考前不易猜测。除此之外, 目前客观测试已有一套完善 的统计分析手段, 如, 项目分析 (item analysis) 、分数等值 (score equating) 处理、题库(item banking) 建设等等。而其 它测试则暂时还缺乏这种现成的手段。所有这些都说明客 观测试的可行性较强, 其对语言教学的影响更不可忽视。 [11]

\section{2 翻译课程客观试题在教学中的反拨效应}

Hughes 在解释反拨作用于教学的工作机制上也作了 一些尝试。他把教学全部内容分为“参与者” (Participants)、 “过程” (Process) 和“结果” (Product) 三部分。[5] “参与 者”指学生、教师、教学管理人员、教材编写人员和出版商, 认为他们对工作的认识和态度可能会受到测试的影响; “过 程”包括参与者所进行的对学习过程产生作用的所有活动, 如材料编写、大纲设计、教学教法的调整、学习方法与考 试策略的应用等; “结果”指学习到的东西和学习的质量, 如知识技能、流利程度等。[3] 综合这三部分, 他建立起一 个测试对教学反拨作用的模型, 认为测试的性质会先影响 参与者对教学活动的认识和态度, 而这种认识和态度又会 影响到参与者在进行活动中的所作所为, 诸如只练习那些 在测试中出现的项目等, 这必然会影响学习结果。因此, 要 提高测试的正面反拨效应, 单从测试方面进行讨论是不够 的, 还应从教和学以及其他方面加以探讨。笔者认为鉴于 客观试题在翻译测试中的上述特点, 其对教学的正面反拨 作用可同样从Hughes的PPT模式探讨。

首先, 针对第一部分“参与者”, 我们重点分析学生与 教师这两个关键角色。客观题型对测试内容的全面覆盖的 能从正面积极地影响学生的对课程的学习态度。在翻译测 试中不难看到,一部分学生由于原来语言功底较好, 对课程 重视不够, 但仍然能够在英汉互译题目中表现不俗。值得 注意的是, 经验告诉我们, 此种“不俗”的表现是阶段性的, 单一的, 即不能代表未来在同龄人中语言驾驭能力的竞争 力, 也不能代表在本学科发展的潜力。另一种现象是主观 试题多取材于某模拟试题或某名篇, 往往会有部分学生恰
巧在以往的学习中遇到过甚至练习过此文的译作, 在此种 情况下, 如该类试题占去了较大分值, 显然就削弱了该测 试的效度。测试中出现的这些片面的结果, 必然会助长部 分学生的盲目自信或依赖运气, 导致其轻视课程教学, 进 而丧失了获得本学科基础理论知识的机会, 阻碍了自己以 后在此领域的发展。多元化形式的客观试题由于能够在最 大限度涵盖本课程内容, 无疑有利于提高学生对课堂教学 的重视程度。

测试对教学影响的第二部分是“过程”。即材料编写、 大纲设计、教学教法的调整、学习方法与考试策略的应用 等。

1993 年J. Charles lderson 和 Dianne Wall 联合在《应 用语言学》 (Applied Linguistics) 杂志上发表了题为《反 拨作用真的存吗?》（Does Washback Exist?）的文章。他 们提出了以下 15 个反拨作用假设（Washback Hypothesis）。 具体是: (1) 测试影响教学; (2) 测试影响学习。据此引出 相关的假设: (3) 测试影响教师教什么; (4) 测试影响教师 怎么教; (5) 测试影响学生学什么; (6) 测试影响学生怎样 学; (7) 测试影响教学进度与顺序; (8) 测试影响学习进度 与顺序; (9) 测试影响教学的广度与深度; (10)测试影响学 习广度与深度; (11) 测试影响教学双方对教学内容、教学 方法的态度; (12) 有重要影响的测试具有反拨作用; (13) 无重要影响的测试没有反拨作用; (14) 测试反作用于所有 学生和教师; (15) 测试只对某些学生和教师产生反拨作用, 对另外的人则没有。[9]

虽然假设有 15 条之多, 但可以看出, $1-10$ 条假设都 反映了测试对教学 “过程” 的影响。材料编写、大纲设计包 括的内容有 “教什么”; 而教学教法的调整、学习方法与考 试策略的应用等则体现了“怎么教”、“怎样学”。

测试对教学影响的第三部分是“结果”, 即学习到的东 西和学习的质量, 如知识技能、流利程度等。毫无疑问, 对理论知识及翻译技巧的测试能够从长远的目标促进学生 在本学科的发展, 如试题容易受偶然因素或教师的主观因 素影响, 便未能对教和学起到反拨效应。

Bailey 在一篇有关反拨效应的文献综述中, 归纳了 提高测试正面反拨效应的四个方面: 语言学习目标、测试 的真实性、学习者的自主性和自我测评、测试结果的反馈, 并在此基础上提出了衡量一个测试是否具有正面反拨效应 的8条标准: 测试参与者是否了解测试的目的和测试结果的 用途? 测试结果的反馈是否清楚、详尽和及时? 测试参与 者是否认为测试结果公正、可信? 测试是否测了课程计划 的教学内容? 测试是否基于明确的目标和目的测试是否基 于正确的理论原则? 测试是否使用了真实的语篇和测试任 务? 测试参与者是否进行了自我评估? [3]这8条标准的大 
部分与Hughes的意见一致, 如“内容覆盖面”、“教学目 标 ”、“对测试的了解 "等。同时在对比中也可以看出, Bailey更加强调了教学内容在测试中的反映和测试结果对 学习过程和学习者的反拨作用。

\section{4. 结束语}

教育需要评价, 没有评价的教育是盲目的教育。考试 应完整体现大纲规定的教学内容。翻译测试是检查教学大 纲执行情况、评估教学质量的一种有效手段, 是获取教学反 馈信息的主要来源和改进教学的重要依据。在测试多项理 论要求和翻译测试现实需求激烈冲突之下, 对试题形式进 行不断的探讨和改进是的必要的。如何能使翻译测试更为 全面客观地考核考生语言应用能力和翻译理论技巧, 如何 能为使翻译测试在教与学方面达到更为正面的反拨效应, 则需要广大外语工作者和教育者进一步探索。

\section{参考文献(References)}

[1] Bachman L F, Palmer A S. Language testing in practice. Oxford: Oxford University Press, 1996,.pp. 254 - 256.

[2] Bachman, L F. Fundamental Considerations in Language Testing. Oxford: Oxford University Press. 1990.

[3] Bailey, K. M. "Working for Wash back: A review of the Wash back Concept in Language Testing," Language Testing, vol.13, no. 3, pp. 257-279, 1996.
[4] The TEM Testing Center, Shanghai International Studies University. The test for English majors (TEM) validation Study. Shanghai: Shanghai Foreign Language Education Press, 1997. p.164.

[5] Hughes A. Testing for Language Teachers. Cambridge: Cambridge University Press, 1989.p. 26.

[6] Syllabus for College English Majors. Shanghai: Shanghai Foreign Language Education Press. 2000.

[7] Mu, Lei. Research on Chinese Translation Teaching. Shanghai: Shanghai Foreign Language Education Press, 2000.p.83.

[8] Huang, Dayong, Yang, Bingjun. An Overview for Backwash Effect of Testing in Foreign Language Teaching. 2002 p. 288-191.

[9] Huang, Jianping. On the Backwash Effect of Language Testing. US-China Foreign Language, 2005(10).p.70-73

[10] Li, Xin. On the "Constructing Validity" of Translation Test and Its Implementation. Northeastern University Journal. 2004(6): p.217-219

[11] Li. Xiaoju. The Science and Art in Language Testing. Changsha: Hunan Education Press, 1999: p.12-53

[12] Yang, Huizhong. On the Validity in Band 4 and Band 6 of College English Test. Shanghai: Shanghai Foreign Language Education Press, 1998:p.55-59. 